\title{
Scottish Otolaryngological Society: abstracts from the November 2005 meeting, Edinburgh, Scotland, UK
}

\section{Our initial experience of day case suction diathermy adenoidectomy}

S Ali, M L Montague, H Kubba

From the Royal Hospital for Sick Children, Yorkhill, UK

\section{Introduction}

Adenoidectomy is one of the most common operations performed in children in the UK. The most common method used is curettage, which is performed blindly and relies on tamponade with a swab for haemostasis. Postoperative haemorrhage is difficult to control. Suction diathermy adenoidectomy under direct vision offers more complete removal of the adenoids, less intra-operative blood loss and significantly less risk of post-operative haemorrhage. We present the first cycle of an audit of suction diathermy adenoidectomy, undertaken with a view to introducing this technique as a day case procedure.

\section{Method}

We prospectively recorded all suction diathermy adenoidectomy procedures performed between January and November 2005. Our usual practice had been to admit all adenoidectomy patients for an overnight stay because of the risk of bleeding. However, children undergoing the suction diathermy procedure were allowed home on the day of surgery whenever the post-operative ward round deemed them fit enough.

\section{Results}

Forty-nine patients were studied in total, 25 boys and 24 girls, ranging in age from two to 17 years (median five years). The most common indication for surgery was persistent otitis media with effusion, and 33 patients received a grommet insertion at the same time as the adenoidectomy. The majority of the operations lasted 20-45 minutes, including anaesthetic time, with two lasting an hour and a half (due to factors unrelated to the procedure). Post-operative observations were carried out at a frequency of half-hourly for four hours, hourly for four hours and four-hourly thereafter (mean 10.5 hours). Thirty-one patients were discharged on the same day as their operation, 17 the next day and one after two days. Four patients' overnight stays were planned pre-operatively because of the child's co-morbidity (e.g. Down syndrome). Fourteen children (29 per cent) who would have been candidates for same-day discharge were kept overnight. This was due to nausea and vomiting in nine, poor oral intake in one and chest infection in another. There were no postoperative bleeding problems and no re-admissions.

\section{Conclusion}

Suction diathermy adenoidectomy offers many advantages, but attention to the problems of post-operative nausea and vomiting are required if we are to realize its full potential as a day case procedure.

\author{
Quality of life after septal surgery \\ N Calder, I Swan \\ From the Glasgow Royal Infirmary, UK
}

Aim

To measure the change in health-related quality of life following septal surgery.

Methods

Glasgow Benefit Inventory (GBI) questionnaires were sent to patients throughout Scotland as part of SENTOS (the Scottish ENT Outcomes Study). In addition, all adult patients undergoing septal surgery in North Glasgow NHS Trust between 1 September 2003 and 28 February 2005 were identified. Any of these patients who had not completed a GBI as part of SENTOS were sent one to complete. The questionnaires were distributed at least six months after surgery.

\section{Results}

A total of 134 GBIs were received. Of these, seven were incomplete and had to be excluded, and a further six were partially complete so only the results for the social and physical subscales could be used. The response rate from SENTOS was 55 per cent. At the time of writing, the response rate from the questionnaires sent (Gartnavel General Hospital patients) was 50.7 per cent (35 out of $69)$. The mean total GBI score was +10.3 , with scores for the general, social and physical subsets being 12.6, 5.2 and 6.8 , respectively.

\section{Discussion}

These scores are much lower than those reported in studies of other interventions. Possible reasons for this are a relatively low morbidity from septal deviation or incorrect indications for surgery.

\section{Conclusion}

Patients reported minimal improvement in health-related quality of life following septal surgery.

\section{Screening for glandular fever in quinsy: is it necessary and cost-effective? An observational study}

M Shareef, B Natarajan

From the Monklands Hospital, Airdrie, UK

Peritonsillar abscess is a common condition encountered in otolaryngological practice, requiring hospitalization. The routine practice in our unit over the years has been to routinely test all patients with quinsy for the presence of glandular fever, although this association has been mentioned in few case reports. ${ }^{1,2}$

We set out to prove or disprove the association of glandular fever in patients with quinsy. We conducted a partly prospective and partly retrospective observational study 
of all patients admitted to our unit with quinsy over a period of 12 months from October 2004. We gathered prospective data for eight months (from February 2005) and retrospective data for four months (from October 2004).

Only patients with a positive diagnosis of quinsy confirmed by aspiration of frank pus were included in the study. A total of 66 patients, 30 male and 36 female, were included in the study. All these patients were screened for glandular fever, including monospot and peripheral screening and liver function tests.

Our analysis showed only one patient out of 66 to have proven glandular fever on haematological screening. We conclude that there appears to be a very low prevalence of glandular fever in patients with quinsy (around 1.5 per cent). Based on the above conclusion, we suggest that routine screening for glandular fever in patients with quinsy is unnecessary and invasive for the patients and time-consuming and cost-ineffective for the trust.

\section{References}

1 Monem SA, O'Conner PFF, O'Leary . Peritonsillar abscess and infectious mononucleosis: an association or a different presentation of the same condition? Ir Med J 1999;92: $278-80$

2 Hanna BC, McMullan R, Hall SJ. Corticosteroids and peritonsillar abscess formation in infectious mononucleosis. J Laryngol Otol 2004;118:459-61

Sphenopalatine foramen identification and anatomy

L Hadoura, C M Douglas, G McGarry

From the Department of Otolaryngology, North Glasgow Hospitals, Glasgow, UK

\section{Introduction}

The surgical anatomy of the sphenopalatine foramen (SPF) is clinically important as this is where the sphenopalatine artery enters the nose. Endoscopic ligation of the sphenopalatine artery is a recognized treatment for control of intractable posterior epistaxis that cannot be controlled by conventional methods. However, in a bleeding patient, a good knowledge of the anatomy is essential to help identify the SPF and ligate the artery.

\section{Objective}

We investigated the anatomy of the sphenopalatine foramen using an image guidance system (Brain Lab) and human skulls.

\section{Method}

The Brain Lab surgical navigation system was used. Twenty computed tomography (CT) scans of the head and neck of patients being treated for head and neck pathology were used. No patients had a history of SPF artery ligation. The right and left sphenopalatine foramena were identified on each CT scan. Two fixed anatomical landmarks were used to measure the distance from the SPF to the anterior part of the nose. Ten Caucasian skulls obtained from the Glasgow University anatomy department were used as controls. We hope that these measurements will enable easier identification of the sphenopalatine foramen.

\section{Craniofacial resection and the application of topical fluorouracil in the treatment of tumours of the nasal cavity and paranasal sinuses: the Edinburgh experience}

\author{
M R Humphreys \\ From the Western General Hospital, Edinbur, UK
}

\section{Objectives}

This paper aims to report my team's experience of the management of sinonasal malignancy using a regimen of surgical debulking and topical chemotherapy (5-fluorouracil) and also to report our long-term disease-free survival in a small series of patients.

\section{Design and setting}

A review of retrospectively collected data was undertaken within a tertiary cancer centre.

\section{Patients}

I reviewed eight consecutive patients with sinonasal malignancies referred to the department of head and neck surgery, Western General Hospital, Edinburgh, between September 1997 and August 2004.

\section{Interventions}

Patients underwent surgical debulking via anterior craniofacial resection and received repeated applications of topical chemotherapy (5-fluorouracil) and necrotomy. Additionally, one patient received pre-operative chemoand radiotherapy and one patient required post-operative surgery for neck lymph node metastases, in addition to chemo- and radiotherapy.

\section{Main outcome measures}

Main outcome measures comprised clinical complications related to the therapy, evidence of recurrent disease and disease-free survival time.

\section{Results}

There were no peri-operative deaths. One patient required a post-operative dacrocystorhinostomy for persistent epiphoria. Two patients were identified as having recurrent disease. Both patients with recurrence were identified clinically within nine months of their procedure. One patient was noted to have metastatic recurrent disease within four months of the procedure. The average disease-free survival time was 31.4 months. One patient remained disease-free 96 months after surgery.

\section{Conclusions}

I report my team's experience of a small series of patients undergoing a combination of surgical debulking and repeated application of topical chemotherapy (5-fluorouracil) for sinonasal malignancy. I conclude that this form of combination therapy is the treatment of choice in the majority of patients, leading to long-term, disease-free survival.

\section{A combined ENT, audiology and education surveillance clinic for children with Down syndrome: results from our first year}

\section{S K Kang, K Hunter, M McFarlane*, H Kubba}

From the Royal Hospital for Sick Children, Yorkhill, and the *Greater Glasgow Education Authority, Glasgow, UK

\section{Introduction}

In 2000, the Down Syndrome Medical Interest Group produced guidelines for the health surveillance of children and adults with Down syndrome, endorsed by various royal colleges and specialty associations. One of the areas covered was audiology; it was suggested that all children with Down syndrome have hearing testing at birth, then at least once a year until they start school, and once 
every two years thereafter into adulthood. In greater Glasgow, the audiology and education services have for some time conducted a joint clinic for annual hearing surveillance of all pre-school children with Down syndrome. For the past year, an ENT surgeon has also been present. The aim of this initial review was to estimate the prevalence of ENT problems in these children and to also estimate the proportion of children for whom ENT input was required.

\section{Methods}

A prospective database was kept for one year. Data were collected on all children seen, including the clinical status of each child and the outcome of consultation (i.e. list, discharge or review).

\section{Results}

Between September 2004 and August 2005, 42 pre-school (i.e. aged one to five years) children with Down syndrome were sent appointments for the clinic. Six failed to attend despite numerous reminders. Thirty-eight children were seen, 23 girls and 15 boys. Over the course of the year, 14 of the children (37 per cent) were listed for surgery as follows: grommets, two; grommets and adenoidectomy, four; adenotonsillectomy, six; adenotonsillectomy and grommets, one; overnight pulse oximetry only, one. The indications for surgery were hearing concerns due to otitis media with effusion (OME) in seven children and obstructive sleep apnoea in eight (one child had both). In addition, 11 of the children ( 29 per cent) had conditions which did not require surgery but which were being kept under clinic review (hearing concerns in eight, and hearing and breathing concerns in three). Thus, overall, 47 per cent of children had symptomatic OME and 29 per cent had obstructive symptoms. One child had laryngomalacia which resolved without treatment. None had significant sensorineural hearing impairment. Over the year, three children were discharged from the clinic, to be followed up by their school's audiology service.

\section{Conclusion}

The prevalence of ENT problems in these children was high, and surgical intervention was frequently required.

\section{Preliminary comparative study of audiometry and hearing and auditory processing performance scores in children}

P Fivey, S S M Hussain

From the Department of Otolaryngology, Ninewells Hospital and Medical School, Dundee, UK

\section{Objectives}

We aimed to determine whether the children's hearing and auditory processing performance scores (CHAPPSs) were comparable to their pure tone audiometry results. We also aimed to investigate whether the CHAPPS could be a useful tool for assessment of children's hearing when a pure tone audiogram could not be performed.

\section{Design and setting}

The study compared the CHAPPSs with those of pure tone audiometry; it took place within the otolaryngology outpatient department at Ninewells Hospital, Dundee.

\section{Participants}

We included children aged between three and 13 years of age attending the department who had a pure tone audiogram performed as part of their investigation; we also included the parents of these children, who completed the CHAPPS form with the researcher. In total, 20 patients were included in the study.

\section{Main outcome measures}

The main outcome measures were the hearing threshold on air conduction, as determined by pure tone audiometry, and the subject's CHAPPSs.

Results

Of the audiograms and CHAPPSs $(n=20)$ which were completed and analysed, the best correlation was between average binaural hearing deficit from 500 to $4000 \mathrm{~Hz}$ and total CHAPPSs. The correlation between these two variables was investigated with the least squares method and the standard error of this correlation tested with Student's $t$ distribution. The results showed a strong relationship between the two variables inside the 95 per cent confidence interval, and a relationship was proven to exist between the two $(p<0.01)$. Other trends were observed, but the sample size was too small to make these worth investigating.

\section{Conclusions}

The pure tone audiogram results and the CHAPPSs showed a significant relationship in this small sample. A correlation between total CHAPPS and average binaural hearing deficit was shown, but the sample size was too small to draw any great conclusions. Further and more detailed research is indicated into the various trends of the CHAPPS form as they relate to pure tone audiometry.

\section{The use of intra-nasal neomycin in epistaxis: what are we treating?}

D Baring, D Walker

From the Royal Alexandra Hospital, Paisley, and the Southern General Hospital, Glasgow, UK

\section{Introduction}

Epistaxis forms a significant part of the ENT workload. Once treated, patients are commonly prescribed arachis oil with chlorhexidine and neomycin (traded as Naseptin cream (Alliance, San Diego CA, USA)). Neomycin is an aminoglycoside antibiotic which is effective against most gram-negative bacteria (except Pseudomonas aeruginosa) and some gram-positive bacteria (including staphylococci but not streptococci). This study aims to determine whether there are increased nasal bacterial infection rates in adult epistaxis patients which may cause or perpetuate epistaxis, and to investigate the appropriateness of Naseptin use in this situation.

\section{Methods}

Between October 2004 and April 2005, nasal swabs were taken from patients aged over 18 years presenting with epistaxis and from elective cases attending the pre-assessment clinic. In the epistaxis group, patients were excluded if they had a traumatic aetiology or had been treated with either local anaesthetic spray or Naseptin already or if they were successfully packed bilaterally (however, if packs were removed at initial assessment a swab was taken). In the control limb of the study, patients were excluded if they were attending due to rhinological pathology or had an active upper respiratory tract infection. 
Results

Forty-nine patients were entered into the study (control group, $n=23$; epistaxis group, $n=26$ ). Staphylococcus aureus was grown in six patients ( 23 per cent) from the epistaxis group and in five patients ( 21 per cent) from the control group. Coagulase-negative staphylococci were grown in three patients (11 per cent) from the epistaxis group and in eight patients (34 per cent) from the control group. Normal skin flora was grown in three patients (11 per cent) from the epistaxis group and in three patients (13 per cent) from the control group. There was no growth in 13 patients (50 per cent) from the epistaxis group and in seven patients (30 per cent) from the control group.

\section{Discussion}

There was no difference in the bacterial carriage between the two groups, other than a greater number of coagulasenegative staphylococci grown in the control group. The numbers found to have $S$. aureus in the control group fits with previous studies and the numbers of cases with $S$. aureus or normal skin flora were not significantly different between the two groups. There is evidence to suggest that Naseptin is helpful in the management of paediatric epistaxis. There is no similar evidence for its use in adult epistaxis. This problem commonly occurs in an elderly population with other contributing factors such as hypertension or anticoagulation therapy. Our study suggests that there is no increased nasal bacterial carriage in epistaxis patients. Further work is needed to determine whether simple emollient would be as useful in preventing rebleeds.

\section{Infection control in ENT}

T Kunanandam, I Nixon

From the Southern General Hospital, Glasgow, UK

\section{Background}

Dr I P Semmelweis is now recognized as a pioneer of antiseptic policy and prevention of nosocomial disease. His discovery, made in 1847, that puerperal fever was contagious and it could be controlled by employing handwashing measures amongst medical staff, was discredited at the time. At that time, the medical community seemed hostile to the suggestion that it could be implicated in patient morbidity and mortality. It was also argued that hand-washing in between patients was time-consuming. These issues have now resurfaced, particularly with reference to methicillin-resistant Staphylococcus aureus (MRSA), which now attracts extensive media coverage and government focus. Cases of MRSA have been reported since the early 1960s, shortly after Elek and Fleming used a methicillin spray in a nursery environment in an attempt to eradicate $S$. aureus carriage. This led to the development of methicillin-resistant strains, and the reported incidence of MRSA has steadily increased ever since. In modern hospitals, MRSA infection accounts for significant patient morbidity and mortality and hence also impacts on economic factors relating to hospital care. Methicillin-resistant $S$. aureus carriage rates are implicated in many cases of eventual MRSA infection.

\section{MRSA in ENT practice}

The prevalence of MRSA has been reported as 6 per cent in cases of otitis externa (Walshe et al., 2001), 9 per cent in cases of chronic rhinosinusitis (Manarey et al., 2004),
5 per cent in patients undergoing fibre-optic endoscopic sinus surgery (Jiang et al., 1999), 0.2 per cent in patients undergoing tympanostomy tube placement (Hartnick et al., 2000) and 45 per cent in patients undergoing head and neck surgery (Watters et al., 2004).

\section{Departmental audit}

Over a two-month period, we assessed the MRSA carriage rate in elective admissions to the ENT ward at the Southern General Hospital, Glasgow. All patients who agreed to participate were swabbed for MRSA in the nose, throat and groin. Of 116 patients presenting during the study period, 114 entered the study. Patients comprised 48 men (42 per cent) and 66 women (58 per cent) and their ages ranged from 15 to 81 years (mean, 50.2 years). They underwent the following types of surgery: rhinology, 33 (28\%); otology, nine (8\%); general, $10(9 \%)$; and head and neck, $62(54 \%)$.

There was one positive result for MRSA in this study, in an 81-year-old woman with a history of previous hospital admissions and antibiotic use who was admitted for revision dacrocystorhinostomy. One patient who did not wish to participate in the study was later found to have a postoperative infection that was culture-positive for MRSA; notably, he was a carer in a nursing home. Overall, there was a low incidence ( 0.9 per cent) of MRSA carriage in ENT elective surgery patients.

From these results, we conclude that selective screening may be more effective in identifying MRSA carriage in patients. Hospital MRSA acquisition and transmission rates need to be addressed, and this is more likely to be a problem in long-stay and head and neck cancer patients, as suggested in the literature.

\section{Infection control measures}

There are various strategies available for controlling MRSA rates in hospitals.

There is strong evidence to support the use of alcohol hand gels in reducing MRSA transmission. Compliance is thought to be higher with gels compared with traditional hand-washing as the former is less time-consuming. Ideally, these gels should be freely available for use.

Targeted screening programmes to identify patients at risk of carrying MRSA have been shown to be more costeffective and equally sensitive compared with screening all hospital admissions. Identification should then be followed by appropriate treatment and isolation. An argument could also be made that head and neck patients should be segregated from general and elective ENT patients, in whom there is a relatively low MRSA carriage rate; this further reinforces the usefulness of day surgery and short-stay units.

Staff screening is a more problematic area. Eradication measures in this instance have implications for family members and also for the health service through absence of staff from work. Eradicating MRSA carriage in staff has also been shown to be more challenging; tonsillectomy has been performed in two cases for persistent throat carriage.

\section{Future considerations}

Methicillin-resistant $S$. aureus is unfortunately endemic in many countries, including the UK, and total eradication is an unrealistic goal; our focus should be on controlling MRSA rates. Responsible antibiotic prescribing is fundamental in the prevention of further antibiotic resistance and the development of new 'super bugs'. 


\section{Randomized, single-blinded, controlled trial of outcome for coblation versus cold steel tonsillectomy}

\author{
H Jayasinghe, A Williams, A I G Kerr
}

From the Department of ENT, Western General Hospital, Edinburgh, UK

\section{Background}

Coblation is a soft tissue surgical technique used for tonsillectomy. Early published results showed a significant decrease in the amount of post-operative pain experienced by adult patients undergoing coblation tonsillectomy. ${ }^{1,2}$ More recent studies have shown no significant change in outcome from coblation compared with traditional methods. ${ }^{3}$ Coblation tonsillectomy has been reported to cause more intra-operative blood loss than cold steel dissection; ${ }^{4}$ however, other studies have suggested that the risk of intra-operative blood loss for coblation techniques is the same $a s^{5}$ or less than ${ }^{6}$ that for traditional methods.

\section{Study objective}

The aim of this study was to compare post-operative outcomes, in terms of symptoms and complications, of coblation tonsillectomy compared with cold steel dissection. The main outcome measures were operative time, blood loss, post-operative pain and post-operative complications.

\section{Design}

This study included 60 adults scheduled to receive tonsillectomy for recurrent tonsillitis, presenting to the Western General Hospital, Edinburgh. Patients were randomized into two study groups: coblation and cold steel tonsillectomy. A single-blinded technique was adopted. All patients were given a questionnaire with which to document post-operative perception of pain, graded using a visual analogue scale, from day one to day 11. Operative blood loss and complications or re-admissions were also recorded. All statistical analyses were performed using Statistic software.

\section{Results}

Thirty patients (eight men and 22 women) received coblation tonsillectomy and 30 patients (six men and 24 women) received cold steel tonsillectomy. The mean operative time was greater for cold steel tonsillectomy compared with coblation, with means of 23 min (standard deviation (SD) $5.54 \mathrm{~min}$ ) and $14 \mathrm{~min}$ (SD $4.37 \mathrm{~min}$ ), respectively. Estimated blood loss was greater for cold steel tonsillectomy compared with coblation tonsillectomy, with means of $68.9 \mathrm{ml}$ compared with $19.5 \mathrm{ml}$, respectively. Complications included one secondary haemorrhage in the coblation group and one secondary haemorrhage and re-admission in the cold steel tonsillectomy group. Additionally, one patient receiving coblation tonsillectomy developed a uvula haematoma which resolved completely with conservative management. The differences in pain assessment for coblation compared with cold steel were not statistically significant to $p<0.005$. However, the mean pain score was higher for the coblation group, at 5.14 (95 per cent confidence interval (CI) 4.18 6.09; SD 1.36), compared with the cold steel group, at 4.52 (95 per cent CI 3.61 5.44; SD 1.36). The overall rate of secondary haemorrhage was 3.3 per cent $(n=1)$ for both groups, with no reactive haemorrhages reported. Estimated blood loss was greater for cold steel tonsillectomy compared with coblation tonsillectomy, with means of $79.9 \mathrm{ml}$ (SD 62.4) compared with $21.8 \mathrm{ml}$ (SD 25.1), respectively; this was statistically significant to $p=0.0009$. Losses to follow up were significant, with $9 / 30$ (30 per cent) patients in the coblation group and 11/30 (36 per cent) patients in the cold steel tonsillectomy group not responding.

\section{Discussion}

This randomized, single-blinded, controlled study demonstrated no distinct advantage in post-operative recovery for intracapsular coblation tonsillectomy compared with traditional, subcapsular, cold steel tonsillectomy. In fact, in adults, the mean pain score was higher for coblation compared with cold steel tonsillectomy. Differences in surgical time and intra-operative estimated blood loss were statistically significant but clinically insignificant for cold steel compared with coblation tonsillectomy. There were no differences in complication rates in either group. Limitations of the study include small sample size and significant losses to follow up.

\section{References}

1 Timms MS, Temple RH. Coblation tonsillectomy: a double blind randomized controlled study. J Laryngol Otol 2002; 116:450-2

2 Hall DJ, Littlefield PD, Birkmire-Peters DP, Holtel MR. Radiofrequency ablation versus electrocautery in tonsillectomy. Otolaryngol Head Neck Surg 2004;130:300-5

3 Philpott CM, Wild DC, Mehta D, Daniel M, Banerjee AR. A double blinded randomised controlled trial of coblation versus conventional dissection tonsillectomy on post operative symptoms. Clin Otolaryngol 2005;30:477-8

4 Lowe D, van der Meulen J, National Prospective Tonsillectomy Audit. Tonsillectomy technique as a risk factor for postoperative haemorrhage. Lancet 2004:364:697-702

5 Divi V, Benninger M. Postoperative tonsillectomy bleed: coblation versus noncoblation. Laryngoscope 2005;115:31-3

6 Metternich FU, Sagowski C, Wenzel S, Jakel K. Tonsillectomy with the ultrasound activated scalpel. Initial results of technique with Ultracision Harmonic Scalpel. HNO 2001; 49:465-70 